\title{
Short duration Phantosmia changes in a COVID-19 patient in Bangladesh.
}

\author{
Mohammad Ashraful Amin ${ }^{1}$, Sabrina Nahin ${ }^{2}$, Jannatul Fardous ${ }^{3}$, Md Faisal Kabir Rozars ${ }^{4}$, \\ and Mohammad Delwer Hossain Hawlader ${ }^{1}$ \\ ${ }^{1}$ North South University \\ ${ }^{2}$ Green Life Medical College and Hospital \\ ${ }^{3}$ National Institute of Kidney Diseases \& Urology (NIKDU) \\ ${ }^{4}$ Chittagong Medical College
}

February 6, 2022

\begin{abstract}
Phantosmia is the sensation of a fragrance intermittently or continuously when no odorant trigger is available. Without any further complaints, COVID-19 individuals may experience olfactory hallucination. Phantosmia was unnoticed for a long time sinc
\end{abstract}

\section{Introduction}

SARS-CoV-2 infection and COVID-19 disease have been linked to a variety of olfactory disorders, especially anosmia, phantosmia, and dysgeusia (1-4). Phantosmia is a subjective olfactory disorder defined as a perception of odor without an actual activating odor. In the literature, olfactory hallucinations were linked to migraine headaches, endoscopic skull base surgery, intracranial tumors, brain radiation treatment, and head injuries (5). The smell is generally only noticeable for a few minutes the first time it is experienced, and it almost always appears out of nowhere (6). Among the first signs of COVID-19 disease is olfactory impairment, which can manifest as hyposmia, anosmia, or a sudden loss of smell (7). However, there has never been a report of COVID-19-related phantosmia or olfactory hallucination in Bangladesh. COVID-19 patients who had olfactory hallucinations are reported in this paper.

\section{Case Report}

A 32-year-old man physician complained of constantly "smelling onions and garlic" after his one week of COVID-19 diagnosis. On the 7th December 2021, the patient developed a low-grade fever, seemed weak and fatigued and was diagnosed with COVID-19 on 9th December 2021. On August 18th, 2021, he finished his second dosage of the COVID-19 vaccination (Moderna). The patients stated that the mysterious odor perception persisted till December 20, 2021. Even though the food was not made with onion or garlic, the patient smelled onion and garlic odors in it. He also detects these scents in fruits. The patient had difficulties eating due to nausea caused by an overabundance of onion and garlic odors, which was impacting his quality of life. While eating, the patient inquired if the mysterious odors could be detected by anyone nearby, but all other family members denied any onion or garlic odors. He denied having taste disturbances, nasal congestion, a runny nose or post-nasal discharge and he had never had a sinus infection. On physiological parameters, physical examination and the cranial nerves II-Xll were normal, with no facial weakness or asymmetry. The motor examinations of the upper and lower extremities were normal. WBC $7.44 \times 109 / \mathrm{L}$, Hgb $14.6 \mathrm{~g} / \mathrm{dl}$, hematocrit 45.0 percent, and platelet count $272 \times 109 / \mathrm{L}$ were unremarkable in the clinical laboratory. Furthermore, the patients had never previously experienced phantosmia. There was no history 
of chronic disease, chronic drug use, maxillofacial trauma, or surgery. On December 21, 2021, while eating an egg and banana breakfast, the patient observed that the onion and garlic odors had vanished. Since then, he hasn't been getting the unpleasant onion and garlic flavors in everything he eats. He does not take any drugs for his phantosmia, such as carbamazepine, and it goes away within a few days. Because the strange odor dissipated quickly, he decided not to get a CT scan or MRI of the brain.

\section{Discussion}

Phantosmia is an olfactory sensation that occurs when there is no odor source. These phantom odors can be strong or weak, familiar or unfamiliar, and cannot be detected by anyone in the immediate vicinity. Phantosmia was defined as a qualitative disruption of smell function and it was not observed in Bangladesh during the COVID-19 pandemic. Persistent rhinosinusitis, craniofacial injury, cerebrovascular disease, seizure disorders, psychological symptoms, brain radiotherapy, neurologic disorders, iatrogenic causes, and neurologic and neurodegenerative disorders are some of the causes of phantosmia. The conductive or sensorineural underlying mechanism of this specific symptom is still unknown (8). In patients with olfactory disorders, phantosmia has been observed to occur up to $25 \%$ of the population, and the incidence is higher in the elderly than in the younger population (9). However, in our situation, the patient was a young one. COVID-19 patients, on the other hand, frequently report a lack of taste or smell without nasal congestion or discharge $(10,11)$.It was clear that late-onset symptoms like qualitative alterations (phantosmia) can develop. In some situations, phantosmia developed after months of no other symptoms (11). In a population-based survey, Sjolund et al. (12) found that phantom odors were encountered fewer than once a month (54 \%) and that the most commonly reported phantom smell was smokey or burnt (46\%). The odor of onion and garlic was present in our case, which was unusual.

SARS-CoV-2 involves the spike protein, which is found in the angiotensin-converting enzyme (ACE)-2 protein, to attach to a membrane receptor complex and the proteolytic activities of host proteases such as TMPRSS2 to invade cells $(13,14)$. ACE-2 is not generated by olfactory nerve cells or olfactory epithelium mitral cells, according to recent single-cell RNA-sequencing and immunostaining investigations, but it is represented at a substantial level by other supporting cells in the olfactory mucosa, such as sustentacular and microvillar cells $(13,15)$. The Olfactory disorder has also been linked to viral illness of vascular pericytes (which express ACE-2) and/or immunologically vascular destruction in the olfactory epithelium and olfactory bulb; indeed, magnetic resonance microscopy research discovered microvascular injury in the olfactory bulbs of COVID-19 patients (16).

In SARS-CoV-2 positive patients in Europe and America, the prevalence of smell and taste impairment ranges from $18.6 \%$ to $90 \%(17,18)$. In one sub-group study, they discovered that Chinese and Bangladeshi participants were more likely than other South East Asian ethnicities to have Olfactory and Taste Dysfunction (OTD) (19). Unfortunately, treating these disorders is difficult. The use of systemic and local glucocorticoids (20), as well as olfactory therapy (21) and carbamazepine (5), has been shown to be beneficial. However, no data on the efficacy of these strategies in post-COVID-19 STD is currently available. However, due to safety concerns, existing evidence does not support the usual prescription of systemic corticosteroids in this circumstance.

On December 1, 2021, the Omicron variant of COVID 19 was discovered for the first time in Dhaka, Bangladesh. Persons who had completed the whole main immunization series and booster doses using mRNA vaccines for COVID-19 can become infected with Omicron variant illnesses. In this period, the Omicron variant may contribute as new symptoms and kinds of compliance, thus we are also conscious of these unexpected COVID-19 symptoms as the variant change's day by day.

Limitation, there was no endoscopy or rhinoscopy to examine the nasal cavity and evaluate for disorders that could explain phantosmia. Imaging techniques, such as Computed tomography, Magnetic resonance imaging (MRI) and electroencephalogram (EEG) scans, were used to search for problems in the nasal cavity, brain, or nervous system, which were also not performed.

Although phantosmia or olfactory hallucinations have not previously been linked to COVID-19 disease 
commonly, the onset of phantosmia due to olfactory impairment is a known occurrence. Albeit the pathogenic cause is unknown, phantosmia occurred following infection with COVID-19 and subsided after the disease was resolved. Despite the small duration of follow-up, this case report revealed the presence of a novel, unique.

\section{Conclusion}

Clinical assessment of abnormalities in chemical senses during COVID-19 could be difficult. Finally, COVID19-induced phantosmia can last for a short time or a long time. Furthermore, these qualitative changes in scent can take months to appear after full recovery from COVID-19-induced quantitative losses in smell. While investigating these phenomena, further prospective research with larger cohorts is required.

\section{Funding}

This research did not receive any specific grant from funding agencies in the public, commercial, or not-forprofit sectors.

\section{Declaration of competing interest}

The authors declare that they have no competing financial interests or personal relationships that could have appeared to influence the work reported in this paper.

\section{Consent}

The patient's written informed consent for publishing of this case report, as well as images, was acquired.

\section{Author contributions}

The article's first draft was written by MAA and MDH. MAA, SN, MFKR and JF contributed to the literature review and manuscript preparation. All authors contributed to the final version by critically reviewing and editing drafts.

\section{Ethical approval}

The article is about a case study. As a result, our Ethics Committee's consent was not required.

\section{Reference :}

1. D'Ascanio L, Pandolfini M, Cingolani C, Latini G, Gradoni P, Capalbo M, et al. Olfactory dysfunction in COVID-19 patients: prevalence and prognosis for recovering sense of smell. Otolaryngology-Head and Neck Surgery. 2021;164(1):82-6.

2. Harikrishnan P. Etiogenic mechanisms for dysgeusia in SARS-CoV-2 infection. Journal of Craniofacial Surgery. 2021;32(1):e111-e2.

3. Landzberg DR, Bery E, Chico S, Koh S, Weissman B. Wernicke encephalopathy from olfactory dysfunction after COVID-19 infection. The Neurologist. 2021;26(6):274.

4. Xydakis MS, Albers MW, Holbrook EH, Lyon DM, Shih RY, Frasnelli JA, et al. Post-viral effects of COVID-19 in the olfactory system and their implications. The Lancet Neurology. 2021;20(9):753-61.

5. İslek A, Balcı MK. Phantosmia with COVID-19 Related Olfactory Dysfunction: Report of Nine Case. Indian Journal of Otolaryngology and Head \& Neck Surgery. 2021:1-3.

6. Leopold DA, Loehrl TA, Schwob JE. Long-term Follow-up of Surgically Treated Phantosmia. Archives of Otolaryngology-Head \& Neck Surgery. 2002;128(6):642-7.

7. Parma V, Ohla K, Veldhuizen MG, Niv MY, Kelly CE, Bakke AJ, et al. Corrigendum to: More Than Smell-COVID-19 Is Associated With Severe Impairment of Smell, Taste, and Chemesthesis. Chemical Senses. 2021;46. 
8. Parma V, Ohla K, Veldhuizen MG, Niv MY, Kelly CE, Bakke AJ, et al. More than smell-COVID-19 is associated with severe impairment of smell, taste, and chemesthesis. Chemical senses. 2020;45(7):609-22.

9. Saltagi MZ, Rabbani CC, Ting JY, Higgins TS, editors. Management of long-lasting phantosmia: a systematic review. International forum of allergy \& rhinology; 2018: Wiley Online Library.

10. Beltrán-Corbellini A, Chico-Garcia J, Martinez-Poles J, Rodriguez-Jorge F, Natera-Villalba E, GomezCorral J, et al. Acute-onset smell and taste disorders in the context of COVID-19: a pilot multicentre polymerase chain reaction based case-control study. European journal of neurology. 2020;27(9):1738-41.

11. Cocco A, Amami P, Desai A, Voza A, Ferreli F, Albanese A. Neurological features in SARS-CoV-2infected patients with smell and taste disorder. Journal of Neurology. 2021;268(5):1570-2.

12. Sjolund S, Larsson M, Olofsson JK, Seubert J, Laukka EJ. Phantom smells: prevalence and correlates in a population-based sample of older adults. Chemical senses. 2017;42(4):309-18.

13. Chen M, Shen W, Rowan NR, Kulaga H, Hillel A, Ramanathan M, et al. Elevated ACE-2 expression in the olfactory neuroepithelium: implications for anosmia and upper respiratory SARS-CoV-2 entry and replication. European Respiratory Journal. 2020;56(3).

14. Hoffmann M, Kleine-Weber H, Schroeder S, Kruger N, Herrler T, Erichsen S, et al. SARS-CoV-2 cell entry depends on ACE2 and TMPRSS2 and is blocked by a clinically proven protease inhibitor. cell. 2020;181(2):271-80. e8.

15. Brann DH, Tsukahara T, Weinreb C, Lipovsek M, Van den Berge K, Gong B, et al. Non-neuronal expression of SARS-CoV-2 entry genes in the olfactory system suggests mechanisms underlying COVID-19associated anosmia. Science advances. 2020;6(31):eabc5801.

16. Lee M-H, Perl DP, Nair G, Li W, Maric D, Murray H, et al. Microvascular injury in the brains of patients with Covid-19. New England Journal of Medicine. 2021;384(5):481-3.

17. Rojas-Lechuga MJ, Izquierdo-Dominguez A, Chiesa-Estomba C, Calvo-Henriquez C, Villarreal IM, Cuesta-Chasco G, et al. Chemosensory dysfunction in COVID-19 out-patients. European Archives of OtoRhino-Laryngology. 2021;278(3):695-702.

18. Santos REA, da Silva MG, do Monte Silva MCB, Barbosa DAM, do Vale Gomes AL, Galindo LCM, et al. Onset and duration of symptoms of loss of smell/taste in patients with COVID-19: A systematic review. American Journal of Otolaryngology. 2021:102889.

19. Soh SH, See A, Teo NW, Tan HK, Palaniappan G, Lim ML, et al. Prevalence of olfactory and taste dysfunction in COVID-19 patients: a community care facility study. European Archives of Oto-RhinoLaryngology. 2021:1-6.

20. Seo BS, Lee HJ, Mo J-H, Lee CH, Rhee C-S, Kim J-W. Treatment of postviral olfactory loss with glucocorticoids, Ginkgo biloba, and mometasone nasal spray. Archives of Otolaryngology-Head \& Neck Surgery. 2009;135(10):1000-4.

21. Damm M, Pikart LK, Reimann H, Burkert S, Goktas O, Haxel B, et al. Olfactory training is helpful in postinfectious olfactory loss: a randomized, controlled, multicenter study. The Laryngoscope. 2014;124(4):826-31. 